\title{
DETC2021-71064
}

\section{FORWARD KINEMATICS FOR SUSPENDED UNDER-ACTUATED CABLE-DRIVEN PARALLEL ROBOTS: A NEURAL NETWORK APPROACH}

\author{
Utkarsh A. Mishra \\ Mechanical and Industrial Engineering Department \\ Indian Institute of Technology Roorkee \\ Uttarakhand, India 247667 \\ Email: umishra@me.iitr.ac.in
}

\author{
Stéphane Caro* \\ CNRS, Laboratoire des Sciences du Numérique de Nantes \\ UMR CNRS 6004, \\ 1, rue de la Noë, 44321 Nantes, France \\ Email: stephane.caro@Is2n.fr
}

\begin{abstract}
Kinematic analysis of under-constrained Cable-Driven Parallel Robots has been a topic of interest because of the inherent coupling between the loop-closure and static equilibrium equations. The paper proposes an unsupervised neural network algorithm to perform real-time forward geometrico-static analysis of such robots in a suspended configuration under the action of gravity. The formulation determines a non-linear function approximation to model the problem and proves to be efficient in solving for consecutive and close waypoints in a path. The methodology is applied on a six-degree-of-freedom (6-DOF) spatial under-constrained suspended cable-driven parallel robot. Specific comparison results to show the effectiveness of the proposed method in tracking a given path and degree of constraint satisfaction are presented against the results obtained from nonlinear least-square optimization.
\end{abstract}

\section{INTRODUCTION}

Cable-Driven Parallel Robots (CDPRs) are a particular class of parallel robots whose moving-platform (MP) is connected to the robot fixed base frame by a number of cables. Hereafter, the connection points between the cables and the base frame will be referred to as exit points. Such a design brings advantages of low inertia, high payload to weight ratio, and a significantly large workspace as compared to their serial and parallel counterparts.

\footnotetext{
*Address all correspondence to this author.
}

Numerous applications of such robots have been demonstrated, for instance, large scale 3D printing [1], rescue robots [2], large scale telescopes [3], in rehabilitation mechanisms [4] and transfer robots for the elderly [5].

The kinematic analysis for CDPRs proves to be more complex as compared to the classical parallel robots with rigid links. This is generally driven by the fact that cables can only apply unilateral forces, i.e., only pull the MP and not push it, making it fairly difficult to account for static equilibrium. Many researchers have extensively contributed to the kinematic analysis of such robots. The cable lengths tend to be the most feasible proprioceptive measurement for such robots as compared to other physical quantities like cable tensions or orientations. There has been considerable work in including angular sensors [6], camera-based pose estimations [7-10] and tension sensors [11-14]. However, such incorporations come at their own cost of assembly and accuracy. If only cable lengths, i.e., pulley motor position measurements, are considered, the kinematic analysis is significantly influenced by the cable model used. Cables can be considered mass-less and non-elastic, mass-less but elastic, or with non-negligible mass and elastic [15]. For simplicity and introduction of a novel algorithm to solve the Direct Geometrico-Static Problem (DGSP) of CDPRs, this paper considers mass-less, non-elastic cables and suspended CDPRs.

Apart from the nature of cables and the robot configuration, the number of cables modulating the intended degrees of freedom (DoFs) plays a vital role in influencing the system's kinematic 
analysis. Fully-constrained configurations consist of the same number of cables as the degrees of freedom, the external wrench being the final virtual cable to guarantee tautness of all the cables [16]. However, with the reduction in the number of cables, the system becomes under constrained. Under-constrained CDPRs typically have $n$-DoFs controlled by $m(<n)$ cables. Thus, allowing only $m$ DoFs to be controlled. The kinematic analysis of such robots is inherently coupled with static analysis and cannot be solved without solving the combined kinetostatic problem [17]. For such systems, even when the cable lengths are fixed, the MP still moves and adjusts according to the external wrench. Accordingly, the geometrico-static [18] problem becomes more complex and may have several solutions.

The forward kinematics analysis for under constrained suspended CDPRs has been approached as finding all the possible solutions to the geometrico-static equations or incorporating various iterative strategies to solve in real-time. Interval analysis $[19,20]$ is one possible approach to solve for the complete analysis taking into account that some cables may be slack and have been explored for underconstrained systems. Various works have led to optimization problems for real-time analysis based on minimizing the potential energy [21], finding the lowest equilibrium pose [22] or minimizing cable tensions [23]. Iterative (usually, Levenberg-Marquadt) algorithms have found themselves useful in locally minimizing pose errors [24], using Hessian matrix to construct convex problem [21] and Jacobian matrix to solve the linearized approximation of the FK problem [25] at hand.

The optimization problem discussed for the real-time analysis can be seen to be a highly non-linear system and can be easily correlated to neural network architecture. Artificial Neural Network (ANN) has been used extensively in recent research to solve the inverse analysis of serial robots and forward analysis of parallel robots. Supervised methods are employed by collecting ground truth data and training to find a function approximation. Such practices have been extensively applied to serial [26], and cable [27] robots As most controllers directly work on joint space applying direct control over motor winches to modulate cable lengths, path planning in joint space [28] is a better option. In such a situation, processing Cartesian poses for accurate tracking of a planned path can take advantage of a real-time forward kinematics module.

In this paper, an unsupervised neural network algorithm based on real-time forward kinematics of under-constrained suspended CDPRs is presented for a spatial (6 DoFs, 4 cables) configuration. The algorithm is made suitable for trajectory tracking by using the unsupervised neural network weight adaptation framework and assuming that the difference between two consecutive waypoints does not require significant weight updates. Thus, the convergence turns out to be slower for the initial point and faster for the waypoints as compared to the popular non-linear least-square (lsqnonlin by $\mathrm{R}$ MATLAB) optimization framework. Finally, a pre-planned path is tracked and overall performance for the proposed algorithm and lsqnonlin is compared based on the minimization efficiency of the surrogate objective and magnitude of static equilibrium constraint satisfaction.

The model of the manipulator and the associated nomenclature are discussed in Section 2 followed by the description of the various components of the geometrico-static problem in Section 3. The unsupervised neural network formulation is demonstrated in Section 4. Section 5 gives the simulation results obtained using the proposed approach.

\section{PARAMETRIZATION}

Let us consider an $n$-DOF CDPR with $m$ cables. Its $i$ th closed-loop is represented in Fig. 1. The frame $\mathscr{F}_{b}$ of origin $O$ is attached to the base. The frame $\mathscr{F}_{p}$ of origin $P$ is attached to the Moving-Platform (MP). $\mathbf{a}_{i}$ denotes the Cartesian coordinates vector of exit point $A_{i}$ expressed in $\mathscr{F}_{b} . \mathbf{b}_{i}$ denotes the Cartesian coordinates vector of anchor point $B_{i}$ expressed in $\mathscr{F}_{p}$. The MP pose is defined by the vector p pointing from $O$ to $P$ and the rotation matrix ${ }^{b} \mathbf{R}_{p} \in S O(3)$ from $\mathscr{F}_{b}$ to $\mathscr{F}_{p}$.

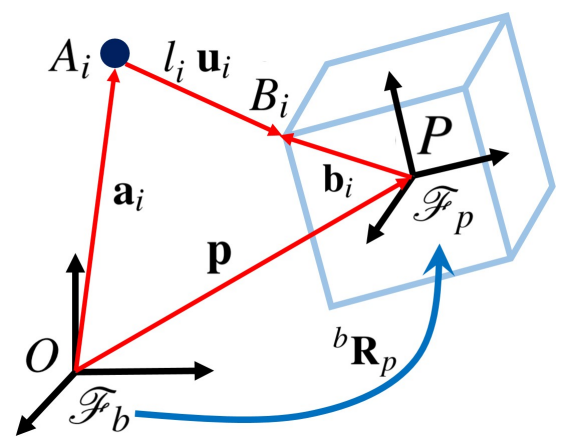

FIGURE 1: $i$ th CLOSED-LOOP OF THE CDPR

The $i$ th loop closure equation is expressed as:

$$
{ }^{b} \mathbf{a}_{i}+l_{i}^{b} \mathbf{u}_{i}-{ }^{b} \mathbf{R}_{p}{ }^{p} \mathbf{b}_{i}-{ }^{b} \mathbf{p}=\mathbf{0}_{3}
$$

$\mathbf{u}_{i}$ is the unit vector along the $i$ th cable pointing from $A_{i}$ to $B_{i}$ and $\mathbf{0}_{\mathbf{3}}$ is a three dimensional zero vector. The subscript ${ }^{b}$ $(p$, resp.) means that the corresponding vector is expressed in frame $\mathscr{F}_{b}$ ( $\mathscr{F}_{p}$, resp.).

The upper and lower bounds on cable tensions are denoted as $\bar{t}$ and $t$, respectively $(\bar{t}=86 \mathrm{~N}, \underline{t}=1 \mathrm{~N})$. The pre-planned path used for the verification purposes are chosen to be within the wrench feasible workspace of the robot (based on CRAFT [29-31]). The MP mass is named $m_{E}$. 


\section{GEOMETRICO-STATIC MODELING}

\subsection{Inverse Kinematics}

The inverse kinematics (IK) formulation for CDPRs is the mapping from the Cartesian space to the cable space. For a given pose and orientation of the $n$-DOF CDPR, IK computes the lengths of the $m$ cables. The main variables and parameters of the kinematics is already described in Fig. 1.

The MP pose, $\mathbf{s}=\left[{ }^{b} \mathbf{p},{ }^{b} \varphi\right]$, is given by the position vector of the center of mass of the moving platform ${ }^{b} \mathbf{p}$ and its orientation with respect to the base frame ${ }^{b} \varphi=[\phi, \theta, \psi]$. Thus, ${ }^{b} \mathbf{R}_{p}=\mathbf{R}_{z}(\psi) \mathbf{R}_{y}(\boldsymbol{\theta}) \mathbf{R}_{x}(\phi)$ and from Eq. (1),

$$
\begin{gathered}
l_{i}^{b} \mathbf{u}_{i}={ }^{b} \mathbf{p}+{ }^{b} \mathbf{R}_{p}{ }^{p} \mathbf{b}_{i}-{ }^{b} \mathbf{a}_{i} \\
l_{i}=\left\|{ }^{b} \mathbf{p}+{ }^{b} \mathbf{R}_{p}{ }^{p} \mathbf{b}_{i}-{ }^{b} \mathbf{a}_{i}\right\|_{2}
\end{gathered}
$$

where $\boldsymbol{l}=\left[l_{1}, l_{2}, \ldots, l_{m}\right]$ is the cable length vector and $\|\cdot\|_{2}$ denoting the two-norm of a vector.. The cable elongations due to elasticity are neglected. This IK mapping from $\mathbf{s}$ to $\boldsymbol{l}$ is referred to the mapping function defined as,

$$
\xi: \mathbf{s} \in \mathbb{R}^{n} \mapsto \boldsymbol{l} \in \mathbb{R}^{m}
$$

\subsection{Forward Kinematics}

The forward kinematics (FK) of the cable-driven manipulators consists of obtaining the platform pose, $\mathbf{s}$ based on a given joint configuration as a set of cable lengths $\boldsymbol{l}$. For the under-actuated case in the paper, the kinematic model is underdetermined. The FK problem is set up as a minimization problem where the relative error between the given cable lengths, $\hat{l}$, and the lengths obtained from the IK of the current pose, $\boldsymbol{l}=\boldsymbol{\xi}(\mathbf{s})$ is a minimum. The error required to be minimized is given by:

$$
e(\mathbf{s})=\|\hat{l}-\xi(\mathbf{s})\|_{2}
$$

Thus, for a given $\hat{\boldsymbol{l}}$, the FK formulation is a function mapping defined by

$$
\begin{gathered}
\zeta: \hat{\boldsymbol{l}} \in \mathbb{R}^{m} \mapsto \mathbf{s} \in \mathbb{R}^{n} \\
\text { such that } \\
\zeta(\hat{\boldsymbol{l}})=\arg \min _{\mathbf{s}}\|\hat{\boldsymbol{l}}-\boldsymbol{\xi}(\mathbf{s})\|_{2}
\end{gathered}
$$

\subsection{Static Equilibrium}

Let an external force $\mathbf{f}_{p}$ and moment $\tau_{p}$ be applied on the moving platform. For the moving platform to remain in equilibrium, there must exist a $m$-dimensional vector of cable tensions, $\mathbf{t}=\left[t_{1}, t_{2}, \ldots, t_{m}\right]$, satisfying the Newton-Euler equation given by,

$$
-\mathbf{W} \mathbf{t}+\mathbf{w}_{\mathbf{e}}=\mathbf{0}_{n}
$$

where $\mathbf{W}$ is the normalized Wrench matrix of the mechanism at this particular MP pose, given by

$$
\mathbf{W}=\left[\begin{array}{ccc}
{ }^{b} \mathbf{u}_{1} & \cdots & { }^{b} \mathbf{u}_{m} \\
(1 / r)^{b} \mathbf{R}_{p}{ }^{p} \mathbf{b}_{1} \times{ }^{b} \mathbf{u}_{1} & \cdots & (1 / r)^{b} \mathbf{R}_{p}{ }^{p} \mathbf{b}_{m} \times{ }^{b} \mathbf{u}_{m}
\end{array}\right]
$$

and $\mathbf{w}_{e}=\left[\begin{array}{ll}\mathbf{f}_{p}^{T} & (1 / r) \boldsymbol{\tau}_{p}^{T}\end{array}\right]^{T}$ as dimensionless homogenized matrices with the help of a characteristic length $r$ which is defined as: $r^{2}=1 / m \sum_{i=1}^{m}\left\|^{\mathscr{F}} \mathbf{b}_{i}\right\|_{2}^{2}($ refer [32]).

\section{NEURAL NETWORK FORMULATION}

The components of robot kinetostatics play a vital role in defining the solution of the forward kinematics and the feasibility of the solution as a measure of satisfaction of the static equilibrium. Unlike serial robots, solving the forward kinematics is relatively difficult than solving the inverse kinematics for such parallel robots. The proposed neural network (NN) formulation is guided by an unsupervised iterative strategy to solve the surrogate objective of finding a suitable MP pose for given cable lengths.

The complete NN framework is built to take the cable lengths from the trajectory in joint space along with the external wrench vector being applied on the MP. Thus the input vector is given by $\mathbf{x}=\left[\begin{array}{ll}\hat{l}^{T} & \mathbf{w}_{\mathbf{e}}^{T}\end{array}\right]^{T}$. Finally, the framework solves for the MP pose and required cable tensions to maintain the MP static equilibrium. Accordingly, the output vector is given by $\mathbf{y}=\left[\begin{array}{ll}\mathbf{S}^{T} & \mathbf{t}^{T}\end{array}\right]^{T}$. The overall proposed strategy can be visualized as an alternative to solve

$$
\begin{gathered}
N N: \mathbf{x} \in \mathbb{R}^{m+n} \mapsto \mathbf{y} \in \mathbb{R}^{m+n} \\
\text { s.t. for } \hat{\boldsymbol{l}}, \mathbf{w}_{\mathbf{e}} \in \mathbf{x} \text { and } \mathbf{s}, \mathbf{t} \in \mathbf{y} \\
N N(\mathbf{x})=\mathbf{y}=\arg \min _{\mathbf{s}}\|\hat{\boldsymbol{l}}-\xi(\mathbf{s})\|_{2} \\
-\mathbf{W}(\mathbf{s}) \mathbf{t}+\mathbf{w}_{\mathbf{e}}=\mathbf{0}_{n}
\end{gathered}
$$

where $\mathbf{W}(\mathbf{s})$ denotes that the wrench matrix $\mathbf{W}$ is a function of the MP pose $\mathbf{s}$. The above quadratic optimization problem was formulated as a surrogate objective $(\mathscr{L})$ based on penalty formation from the constraints such that

$$
\mathscr{L}\left(\mathbf{y}=\left[\mathbf{s}^{T}, \mathbf{t}^{T}\right]^{T}\right)=\|\hat{\boldsymbol{l}}-\xi(\mathbf{s})\|_{2}^{2}+\mu\left\|-\mathbf{W}(\mathbf{s}) \mathbf{t}+\mathbf{w}_{\mathbf{e}}\right\|_{2}^{2}
$$


However, it can be observed that the summation holds two different measurement units, namely, square meters and Newton, respectively. In order to homogenize the surrogate objective, the characteristic length (meter) and the weight (Newton) of the MP were used respectively. Hence, the homogenized objective is expressed as:

$$
\hat{\mathscr{L}}(\mathbf{y})=\frac{1}{r^{2}}\|\hat{\boldsymbol{l}}-\xi(\mathbf{s})\|_{2}^{2}+\mu\left(\frac{\left\|-\mathbf{W}(\mathbf{s}) \mathbf{t}+\mathbf{w}_{\mathbf{e}}\right\|_{2}}{m_{E} g}\right)^{2}
$$

where $g=9.81 \mathrm{~m} / \mathrm{s}^{2}$ is the acceleration due to gravity. Such a penalty function formulation can be solved using Gradient Descent, Newton or Quasi-Newton method and Trust Region methods.

\subsection{Network Architecture}

The formulated input-output problem is solved by means of an unsupervised neural network scheme where the error is calculated using the inverse kinematic lengths of the predicted MP pose vector. The gradients are calculated based on the Stochastic Gradient Descent (SGD) algorithm and the weights are updated based on those gradients.

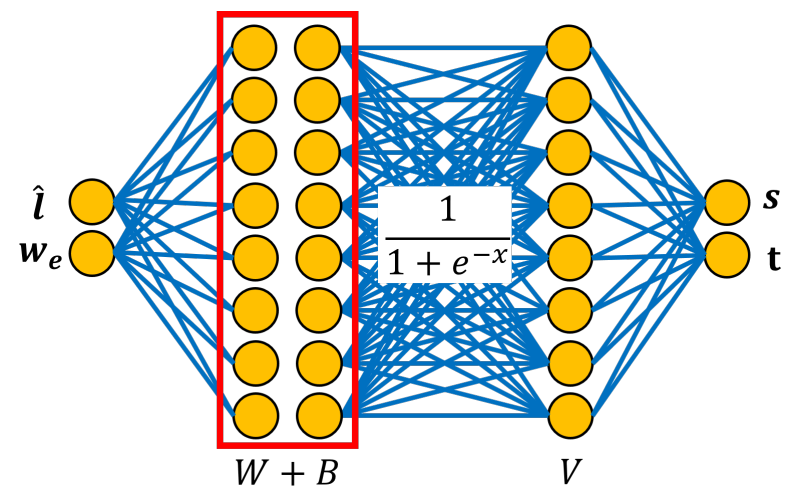

FIGURE 2: CONSIDERED NEURAL NETWORK ARCHITECTURE WITH ACTIVATION FUNCTION

Consider a Neural Network (NN) consisting of two hidden layers with weights $W$ of shape $h \times(m+n), V$ of shape $(m+n) \times$ $h$ and biases $B$ of shape $h \times 1$ respectively, where $h$ is the number of hidden nodes in the hidden layers. The activation functions used for the hidden layers is the sigmoid function, $\sigma(x)$, defined by:

$$
\sigma(x)=\frac{1}{1+e^{-x}}
$$

The back-propagation error is defined using $\hat{\mathscr{L}}$ from Eq. (8). From SGD formulation, the weights and biases are optimized with reference to calculated change in weights $\delta_{W}$ of shape $h \times$ $(m+n), \delta_{V}$ of shape $(m+n) \times h$ and biases $\delta_{B}$ of shape $h \times 1$ given by:

$$
\begin{array}{r}
\delta_{W}(j, i)=\frac{\partial \hat{\mathscr{L}}}{\partial W(j, i)} \quad \forall \quad i=[1(m+n)], \quad j=[1 h] \\
\delta_{V}(k, j)=\frac{\partial \hat{\mathscr{L}}}{\partial V(k, j)} \quad \forall \quad j=[1 h], \quad k=[1(m+n)] \\
\delta_{B}(j, 1)=\frac{\partial \hat{\mathscr{L}}}{\partial B(j, 1)} \quad \forall \quad j=[1 h]
\end{array}
$$

The updated weights and biases $\left(W_{\text {new }}, V_{\text {new }}\right.$ and $\left.B_{\text {new }}\right)$ are obtained by using the above $\delta_{\square}$ values obtained for the corresponding current weights and biases ( $W_{\text {current }}, V_{\text {current }}$ and $B_{\text {current }}$ ). The update equations are given by:

$$
\begin{aligned}
W_{\text {new }} & =W_{\text {current }}-\alpha \delta_{W} \\
V_{\text {new }} & =V_{\text {current }}-\alpha \delta_{V} \\
B_{\text {new }} & =B_{\text {current }}-\alpha \delta_{B}
\end{aligned}
$$

where $\alpha$ is defined as the learning rate, typically in the range of $10^{-4}$.

\subsection{Calculation of Derivatives and Weight Updates}

The final problem gets reduced to calculation of the gradients, i.e, the changes in weights. To solve this problem, some pre-calculations are done to represent the predicted output vector $\mathbf{y}^{n n}$ from the input $\mathbf{x}$, such that,

$$
\mathbf{y}_{(m+n) \times 1}^{n n}=V_{(m+n) \times h} \sigma\left(W_{h \times(m+n)} \mathbf{x}_{(m+n) \times 1}+B_{h \times 1}\right)
$$

Each $u^{\text {th }}$ individual term in the output vector is a function of the components of the input vector:

$$
\mathbf{y}_{u}^{n n}=\sum_{j=1}^{h}\left(V(u, j) \sigma\left(\sum_{i=1}^{m+n} W(j, i) \mathbf{x}(i)+B(j, 1)\right)\right)
$$
form:

As a result, the gradients defined in Eqs. (10) to (12) take the 


$$
\begin{aligned}
\frac{\partial \hat{\mathscr{L}}}{\partial W(j, i)} & =\sum_{u=1}^{m+n} \frac{\partial \hat{\mathscr{L}}}{\partial \mathbf{y}_{u}^{n n}} \frac{\partial \mathbf{y}_{u}^{n n}}{\partial W(j, i)} \\
\frac{\partial \hat{\mathscr{L}}}{\partial V(k, j)} & =\sum_{u=1}^{m+n} \frac{\partial \hat{\mathscr{L}}}{\partial \mathbf{y}_{u}^{n n}} \frac{\partial \mathbf{y}_{u}^{n n}}{\partial V(k, j)} \\
\frac{\partial \hat{\mathscr{L}}}{\partial B(j, 1)} & =\sum_{u=1}^{m+n} \frac{\partial \mathscr{\mathscr { L }}}{\partial \mathbf{y}_{u}^{n n}} \frac{\partial \mathbf{y}_{u}^{n n}}{\partial B(j, 1)}
\end{aligned}
$$

The individual segments can be calculated based on derivative of the error with respect to the outputs and the derivatives of outputs with respect to the weights. Finally, Algorithm 1 describes the Unsupervised Forward Kinematics Neural Network (UFKNN).

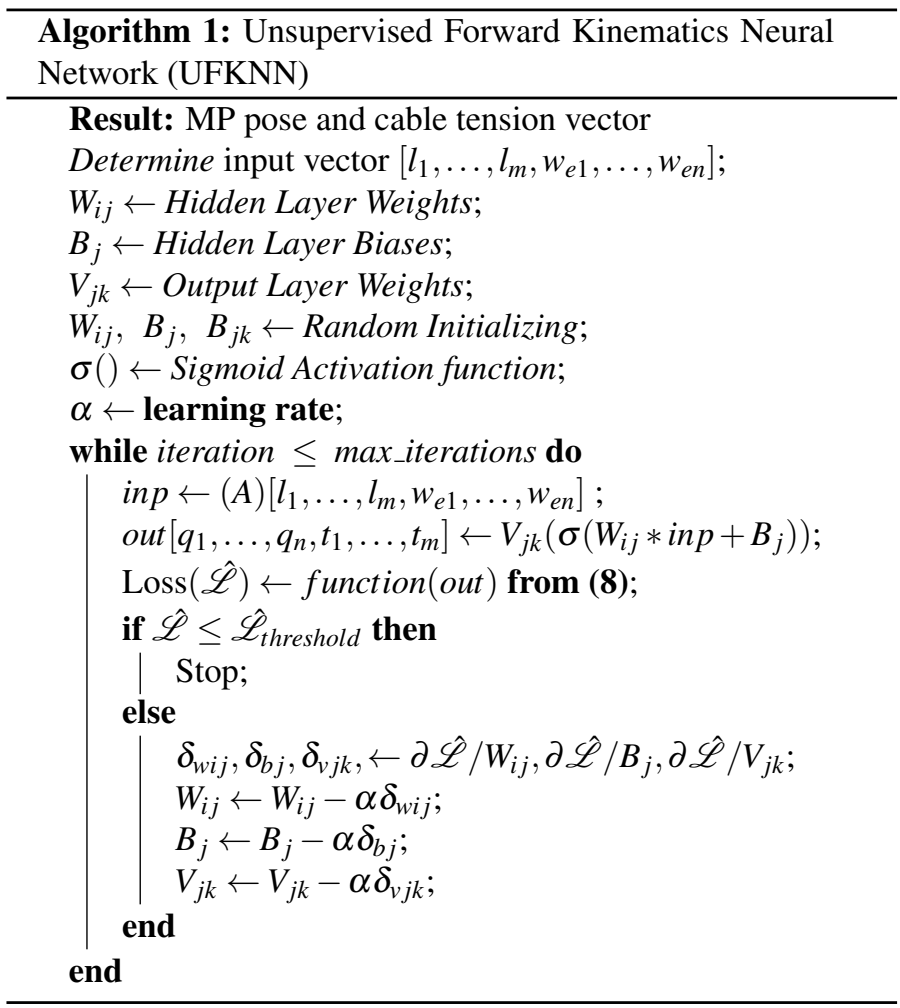

\section{SIMULATION RESULTS}

Algorithm 1 is implemented on a 6-DOF spatial robot with four cables. The primary motivation of the methodology is to develop some form of re-usability by making a module to learn the FK for a particular manipulator about which the module has no clue beforehand. Thus, the results are analyzed by making the manipulator traverse a planned joint space trajectory and are compared against the results obtained with lsqnonlin, a nonlinear optimizer using the Levenberg-Marquardt algorithm. It is expected that the weights estimated for one instance does not vary much when the MP moves to the next waypoint. Hence, the time taken to calculate the forward kinematics for each instance possibly takes less time than the previous one. This conclusively makes trajectory tracking faster by only using proprioceptive sensors. The results are present thereafter considering the rigid cable model.

The spatial 6-DOF suspended underconstrained configuration as shown in Fig. 3a is considered. The setup with a size of $4.24 \mathrm{~m} \times 3.67 \mathrm{~m} \times 2.76 \mathrm{~m}(1 \times \mathrm{b} \times \mathrm{h})$ has a $5.6 \mathrm{Kg}$ moving platform of size $0.28 \mathrm{~m} \times 0.28 \mathrm{~m} \times 0.20 \mathrm{~m}$. As the problem domain increases and becomes more complex, the general intuition that the number of non-linear parameters will increase leads us to choose a higher number of hidden nodes. However, the appropriate learning rate was obtained iteratively by observing the quality of the results. Table 1 gives the parameters that are used in this illustrative example.

TABLE 1: PARAMETERS FOR SPATIAL CDPR-UFKNN ALGORITHM AND LSQNONLIN COMPARISON

\begin{tabular}{cc|cc}
\hline \hline Parameter & Value & Parameter & Value \\
\hline$m_{E}$ & $5.6 \mathrm{Kg}$ & $h$ & 64 \\
$r$ & $0.396 \mathrm{~m}$ & $\alpha$ & $10^{-5}$ \\
$\mu$ & 0.4 & $\hat{\mathscr{L}}_{\text {threshold }}$ & 0.0015 \\
$t_{1}$ & $18 \mathrm{~N}$ & $t_{3}$ & $25 \mathrm{~N}$ \\
$t_{2}$ & $15 \mathrm{~N}$ & $t_{4}$ & $16 \mathrm{~N}$ \\
$\mathrm{itr}_{\text {max }}^{l s q}$ & $2 \times 10^{5}$ & tol $^{l s q}$ & $10^{-100}$ \\
\hline \hline
\end{tabular}

The reference path for this case is chosen to be a vertical spiral in the center of the fixed frame, as in Fig. 3a, and the corresponding path in joint space is shown in Fig. 3b. Such a path considers the extreme points at varying heights. Now, to track the accuracy of the algorithm in finding an appropriate solution, we prepare ground-truth outputs using the Cartesian data and fixed tension vector equal to $\mathbf{t}=\left[t_{1}, t_{2}, t_{3}, t_{4}\right]$, respective values can be seen in Table 1. The wrench to be applied on the MP at every pose instance is calculated by considering the fixed tension vector and the wrench matrix corresponding to that MP pose.

The comparison setup for both the algorithms was based on an iterative rule based on the fact that the right initial solution 


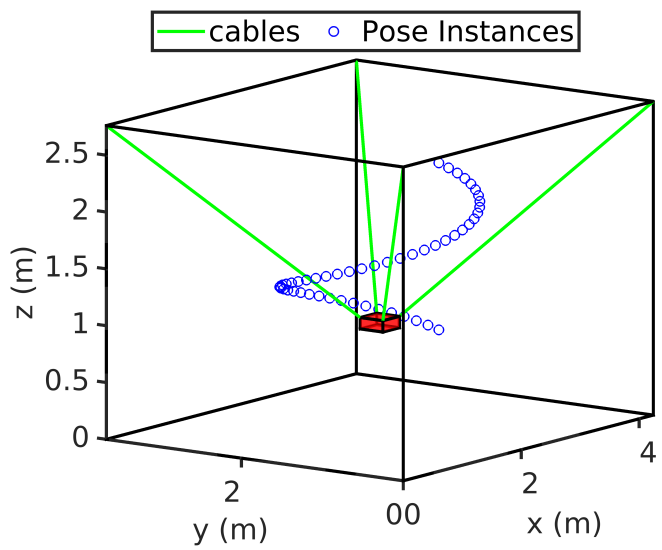

(a) MODEL AND CARTESIAN PATH

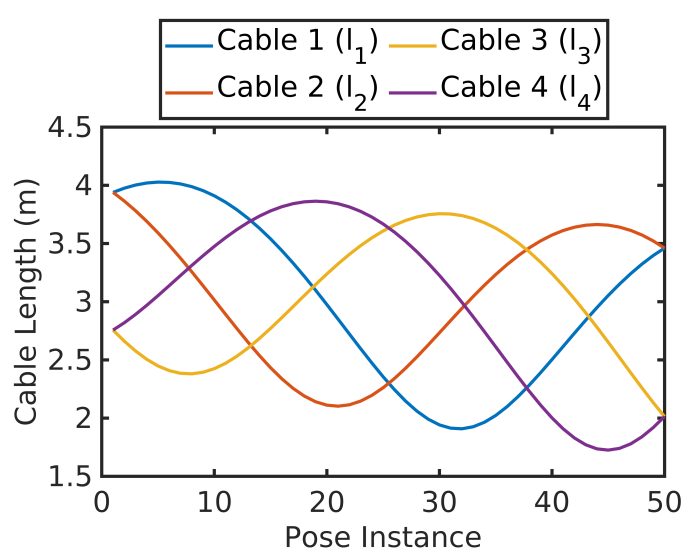

(b) PATH IN CABLE SPACE

FIGURE 3: CDPR CONFIGURATION AND CARTESIAN REFERENCE PATH

should speed up the convergence of the algorithms. The ground truth output belonging to the first pose instance was fed into the algorithms. During this initial setup process, while lsqnonlin takes only 10 secs to converge, the neural network formulation requires about $1 \mathrm{~min}$ to tune the initial network weights. All the calculations were performed using (C)MATLAB with CPU computations on an INTEL @i7-7500U CPU@2.70GHz.

After both the algorithms perfectly fit into the starting pose, the previous outputs from the algorithms were used as initial guesses (for lsqnonlin) or weights (for neural network) for each of the next pose instances. The performance and accuracy are measured on a variety of factors as described below. Here, performance is referred to the overall time and minimization objectives which are the primary optimization objectives. Accuracy, on the other hand, refers to the relative deviation of the cable length, cable tensions and Cartesian poses from the desired values.

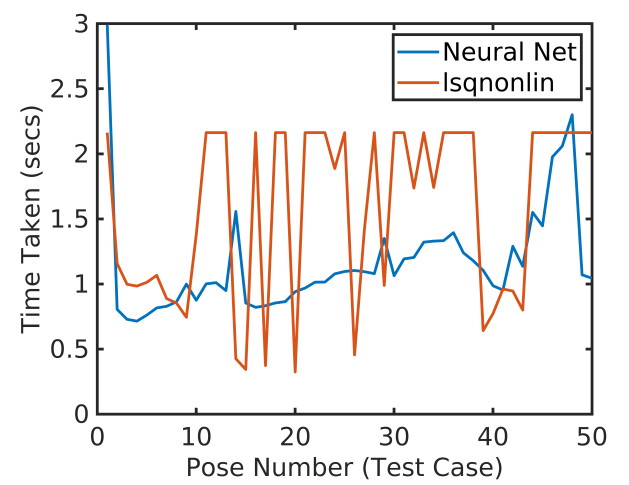

(a) TIME COMPARISON

\subsection{Performance}

The overall performance for both the algorithms was compared based on the computation time for each of the 50 pose instances and the extent of minimization of the surrogate objective. The results are shown in Fig. 4. Figure 4a clearly justifies the learning of the neural network. It should be noted that the preiterations for the initial pose for $1 \mathrm{~min}$ can be considered as the time taken by the algorithm to learn the model-specific kinetostatics. The time taken by the counterpart is relatively higher, which quantitatively corresponds to twice on average. Though lsqnonlin gets the privilege of having a very close initial guess (as the waypoints are very close to each other), it has to perform a black-box optimization every time due to its model-free approach. The UFKNN algorithm does not store the guesses but a non-linear approximation of the kineto-static model itself. This model-based behavior plays a vital role in this advantage.

The minimization of the loss function was clipped at the

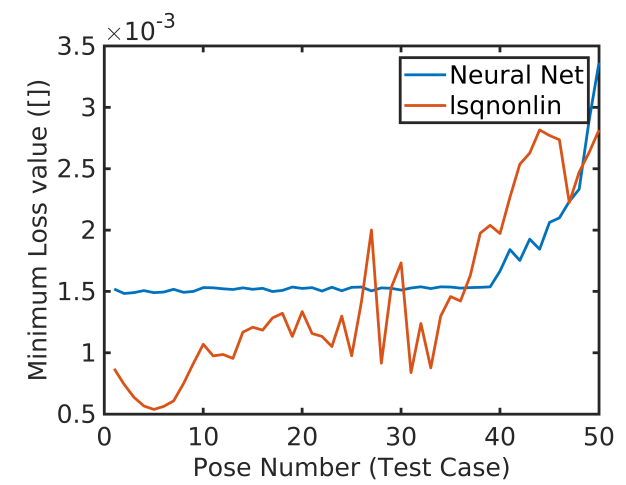

(b) MINIMUM SURROGATE OBJECTIVE VALUE

FIGURE 4: COMPARISON OF OPTIMIZATION OBJECTIVES 


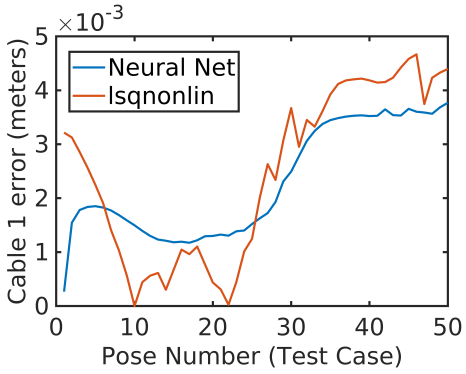

(a) CABLE 1 LENGTH ERROR

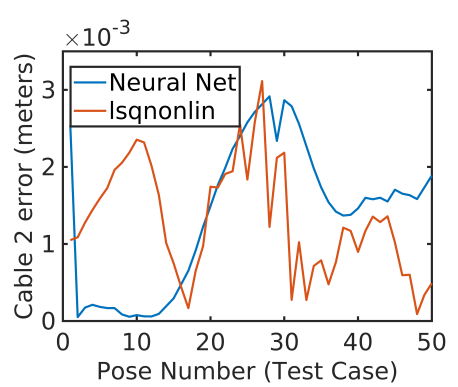

(b) CABLE 2 LENGTH ERROR

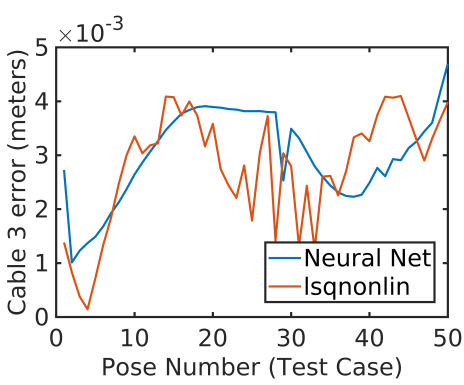

(c) CABLE 3 LENGTH ERROR

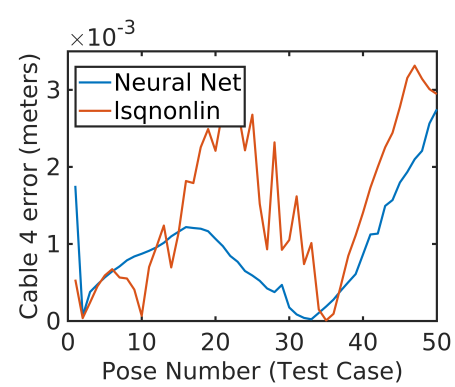

(d) CABLE 4 LENGTH ERROR

FIGURE 5: COMPARISON OF CABLE LENGTH ERRORS FOR SPATIAL CDPR

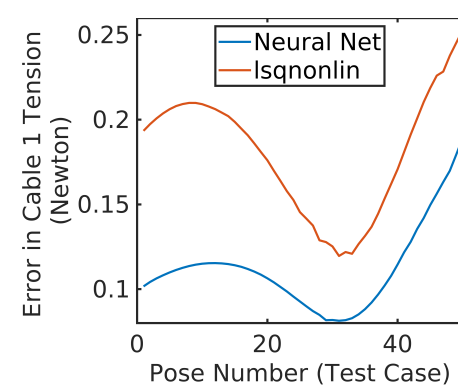

(a) CABLE 1 TENSION ERROR

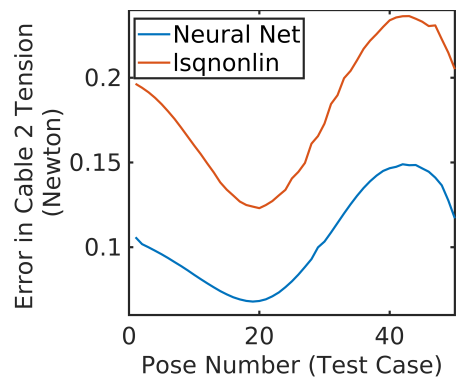

(b) CABLE 2 TENSION ERROR

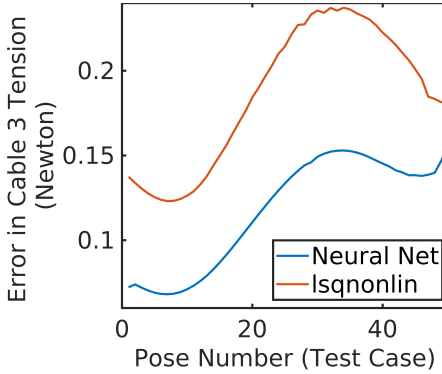

(c) CABLE 3 TENSION ERROR

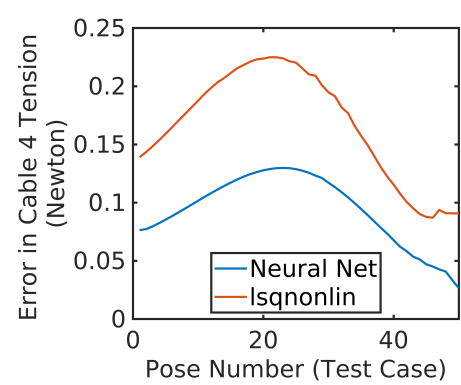

(d) CABLE 4 TENSION ERROR

FIGURE 6: COMPARISON OF TENSION ERRORS FOR SPATIAL CDPR

$\hat{\mathscr{L}}_{\text {threshold }}$ value for the UFKNN algorithm, and hence the values less than that cannot be shown (even if they might be possible). This threshold value was chosen such that the algorithm marginally reaches the optimization performance of lsqnonlin in lesser time. Figure $4 \mathrm{~b}$ shows the negligible difference between the minimized objective values for both the algorithms.

\subsection{Accuracy}

As the problem at hand is a coupling of the kinematics and statics of the under-constrained CDPR model, the pose can be changed to satisfy wrench feasibility and decrease the wrench satisfaction error. Thus, it is essential to judge the formulation's accuracy by comparing it with the ground truth reference poses. The metrics for comparison are the cable lengths, cable tensions, and the predicted Cartesian poses. Figures 5, 6 and 7 cumulatively show the accuracy comparison of both the algorithms.

5.2.1 Cable Lengths The desired cable lengths corresponding to the prescribed path in cable space are a part of the inputs that are given to the algorithms. After predicting the MP pose by the UFKNN algorithm, the obtained cable lengths are calculated through inverse kinematics. The final error is the difference between the calculated and the desired cable lengths. The comparison of these cable errors is given in Fig. 5 for all ca- bles. The maximum deviation in cable length is equal to $5 \mathrm{~mm}$, which is negligible with respect to four-meter frame length. It is apparent that UFKNN reaches the same accuracy as lsqnonlin in terms of cable lengths, but in lesser time.

5.2.2 Cable Tensions The cable tension values were fixed for all MP pose instances, whereas the external wrench, which is also an input to the algorithm, is varied such that the wrench feasibility condition holds. While this is not possible for realization in practical experiments, such conditions can be used to validate the algorithm, which is the main goal of the paper. The outputs of the algorithm correspond to the MP pose and cable tensions. The obtained tensions are compared with the fixed desired tensions, and the error plot is shown in Fig. 6. The maximum absolute error in cable tensions is $0.25 \mathrm{~N}$ for lsqnonlin and $0.20 \mathrm{~N}$ for the UFKNN algorithm. The corresponding mean errors are $0.17 \mathrm{~N}$ and $0.10 \mathrm{~N}$ respectively. The error plot follows a common pattern which might be because of the fixed tension values for all MP pose instances. All these cable tensions usually result in a very high degree of wrench satisfaction i.e. $\frac{\left\|-\mathbf{W}(\mathbf{s}) \mathbf{t}+\mathbf{w}_{\mathbf{e}}\right\|_{2}}{m_{E} g r} \approx 10^{-3}$.

5.2.3 Predicted MP Poses To complete the comparison analysis, the relative deviation of the predicted MP pose from 


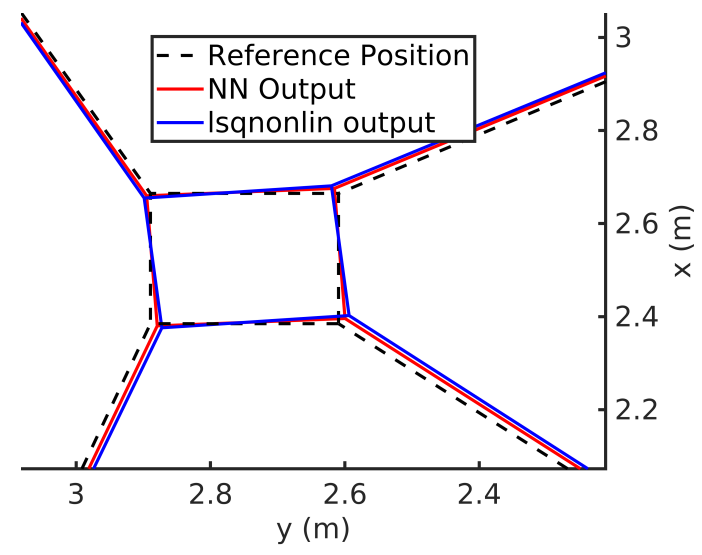

(a) AT $10^{\mathrm{TH}}$ MP POSE

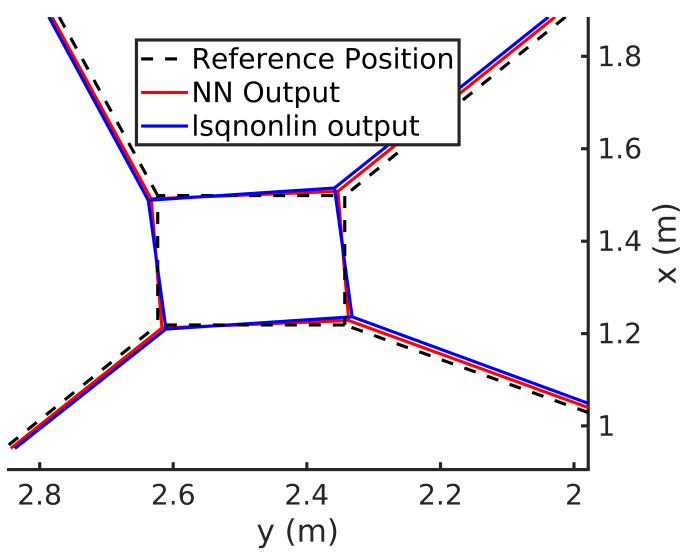

(b) AT $20^{\mathrm{TH}}$ MP POSE

FIGURE 7: COMPARISON OF PREDICTED MP POSES AT TWO INSTANTS

the reference poses is shown in Fig. 7 for two MP poses. The figure is intentionally focused on the MP and zoomed to an extent such that the MP pose deviations tend to be visible. The predicted poses are considerably accurate; however, if observed closely, the relative deviation caused by the UFKNN algorithm is less than that of lsqnonlin. The Cartesian errors for the predicted MP pose by UFKNN for Fig. 7a and Fig. $7 \mathrm{~b}$ are $4.2 \mathrm{~mm}$ and $4.4 \mathrm{~mm}$, respectively, whereas errors for the same MP pose instants as obtained by lsqnonlin are $5.3 \mathrm{~mm}$ and $4.9 \mathrm{~mm}$, respectively. The mean Cartesian error for the MP poses of the complete path is $5.7 \mathrm{~mm}$ for UFKNN algorithm and $6.7 \mathrm{~mm}$ for lsqnonlin. The deviations are mostly due to angular displacements, which are desired to be at 0 degrees, but such high precision cannot be expected from numerical computations.

\section{CONCLUSION}

This paper presented an unsupervised neural network weight adaptation framework according to the kineto-statics of underconstrained Cable-Diven Parallel Robots (CDPRs) and solves the FK problem for a suspended configuration. The approach is validated with simulated results in a 6-DOF spatial CDPR setup and compared with a non-linear least-square optimization-based lsqnonlin algorithm. The comparison demonstrates the advantages of the model-based UFKNN algorithm to approximate the robot's kineto-statics compared with model-free black-box optimization techniques currently in practice. The proposed algorithm can be implemented for near real-time FK. This is particularly beneficial to track paths in cable space or the actuation space. The method is computation time-efficient and works at the same accuracy as other related optimization techniques. Future work includes implementing the proposed algorithm on hardware and conduct simulated experiments to fully leverage its capabilities. Finally, the algorithm will be extended to elastic and sagging-based cable models to determine the solutions to the forward kinematics of more complex and realistic CDPRs.

\section{ACKNOWLEDGMENT}

This work was supported by the ANR CRAFT project, grant ANR-18-CE10-0004, https://anr.fr/Project-ANR-18-CE10-0004

\section{REFERENCES}

[1] Barnett, E., and Gosselin, C., 2015. "Large-scale 3d printing with a cable-suspended robot". Additive Manufacturing, 7, pp. 27-44.

[2] Merlet, J.-P., and Daney, D., 2010. "A portable, modular parallel wire crane for rescue operations". In 2010 IEEE International Conference on Robotics and Automation, pp. 2834-2839.

[3] Su, Y. X., Duan, B. Y., Nan, R. D., and Peng, B., 2001. "Development of a large parallel-cable manipulator for the feed-supporting system of a next-generation large radio telescope". Journal of Robotic Systems, 18(11), pp. 633643.

[4] Homma, K., Fukuda, O., Sugawara, J., Nagata, Y., and Usuba, M., 2003. "A wire-driven leg rehabilitation system: development of a 4-dof experimental system". In Proceedings 2003 IEEE/ASME International Conference on Advanced Intelligent Mechatronics (AIM 2003), Vol. 2, pp. 908-913 vol.2.

[5] Merlet, J.-P., 2010. "Marionet, a family of modular wiredriven parallel robots". In Advances in Robot Kinematics: Motion in Man and Machine, J. Lenarcic and M. M. Stanisic, eds., Springer Netherlands, pp. 53-61.

[6] Garant, X., Campeau-Lecours, A., Cardou, P., and Gosselin, C., 2018. "Improving the forward kinematics of 
cable-driven parallel robots through cable angle sensors". In Cable-Driven Parallel Robots, C. Gosselin, P. Cardou, T. Bruckmann, and A. Pott, eds., Springer International Publishing, pp. 167-179.

[7] Dallej, T., Gouttefarde, M., Andreff, N., Herv, P.-E., and Martinet, P., 2019. "Modeling and vision-based control of large-dimension cable-driven parallel robots using a multiple-camera setup". Mechatronics, 61, pp. 20-36.

[8] Zake, Z., Chaumette, F., Pedemonte, N., and Caro, S., 2020. "Robust 2 1/2D Visual Servoing of a Cable-Driven Parallel Robot Thanks to Trajectory Tracking”. IEEE Robotics and Automation Letters, 5(2), Jan., pp. 660-667.

[9] Zake, Z., Chaumette, F., Pedemonte, N., and Caro, S., 2019. "Vision-Based Control and Stability Analysis of a CableDriven Parallel Robot". IEEE Robotics and Automation Letters, 4(2), Feb., pp. 1029-1036. (also presented ar ICRA'19).

[10] Zake, Z., Caro, S., Suarez Roos, A., Chaumette, F., and Pedemonte, N., 2019. "Stability Analysis of Pose-Based Visual Servoing Control of Cable-Driven Parallel Robots". In CableCon 2019 - Fourth International Conference on Cable-Driven Parallel Robots, pp. 1-12.

[11] Zake, Z., Chaumette, F., Pedemonte, N., and Caro, S., 2021. "Visual Servoing of Cable-Driven Parallel Robots with Tension Management”. In ICRA 2021 - IEEE International Conference on Robotics and Automation, IEEE, pp. 1-8.

[12] Picard, E., Caro, S., Claveau, F., and Plestan, F., 2018. "Pulleys and Force Sensors Influence on Payload Estimation of Cable-Driven Parallel Robots". In 2018 IEEE/RSJ International Conference on Intelligent Robots and Systems (IROS 2018).

[13] Picard, E., Caro, S., Plestan, F., and Claveau, F., 2018. "Control Solution for a Cable-Driven Parallel Robot with Highly Variable Payload”. In ASME 2018 International Design Engineering Technical Conferences and Computers and Information in Engineering Conference IDETC/CIE 2018, no. DETC2018-85304, pp. 1429-1436.

[14] Rasheed, T., Long, P., and Caro, S., 2020. "WrenchFeasible Workspace of Mobile Cable-Driven Parallel Robots". Journal of Mechanisms and Robotics, 12(3), Jan., p. 031009.

[15] Chawla, I., Pathak, P., Notash, L., Samantaray, A., Li, Q., and Sharma, U., 2021. "Effect of selection criterion on the kineto-static solution of a redundant cable-driven parallel robot considering cable mass and elasticity". Mechanism and Machine Theory, 156, p. 104175.

[16] Behzadipour, S., and Khajepour, A., 2005. "A new cablebased parallel robot with three degrees of freedom". Multibody System Dynamics, 13(4), May, pp. 371-383.

[17] Carricato, M., and Merlet, J.-P., 2013. "Stability analysis of underconstrained cable-driven parallel robots". IEEE
Transactions on Robotics, 29(1), pp. 288-296.

[18] Carricato, M., 2013. "Direct geometrico-static problem of underconstrained cable-driven parallel robots with three cables". Journal of Mechanisms and Robotics, 5(3).

[19] Berti, A., Merlet, J.-P., and Carricato, M., 2016. "Solving the direct geometrico-static problem of underconstrained cable-driven parallel robots by interval analysis". The International Journal of Robotics Research, 35(6), pp. 723739.

[20] Merlet, J. P., 2004. "Solving the forward kinematics of a gough-type parallel manipulator with interval analysis". The International Journal of Robotics Research, 23(3), pp. 221-235.

[21] Pott, A., and Schmidt, V., 2015. "On the forward kinematics of cable-driven parallel robots". In 2015 IEEE/RSJ International Conference on Intelligent Robots and Systems (IROS), pp. 3182-3187.

[22] Collard, J.-F., and Cardou, P., 2013. "Computing the lowest equilibrium pose of a cable-suspended rigid body". Optimization and Engineering, 14(3), Sep, pp. 457-476.

[23] Miermeister, P., Kraus, W., and Pott, A., 2013. Differential Kinematics for Calibration, System Investigation, and Force Based Forward Kinematics of Cable-Driven Parallel Robots. Springer Berlin Heidelberg, Berlin, Heidelberg, pp. 319-333.

[24] Pott, A., 2012. "Influence of pulley kinematics on cabledriven parallel robots". In Latest Advances in Robot Kinematics, J. Lenarcic and M. Husty, eds., Springer Netherlands, pp. 197-204.

[25] Santos, J. C., and Gouttefarde, M., 2021. "A real-time capable forward kinematics algorithm for cable-driven parallel robots considering pulley kinematics". In Advances in Robot Kinematics 2020, J. Lenarčič and B. Siciliano, eds., Springer International Publishing, pp. 199-208.

[26] Tang, H., and Notash, L., 2021. "Neural Network-Based Transfer Learning of Manipulator Inverse Displacement Analysis". Journal of Mechanisms and Robotics, 13(3), 04. 035004.

[27] Notash, L., 2020. "Artificial neural network prediction of deflection maps for cable-driven robots". In Proceedings of the ASME 2020 International Design Engineering Technical Conferences and Computers and Information in Engineering Conference (IDETC-CIE2020), Vol. 10: 44th Mechanisms and Robotics Conference (MR).

[28] Mishra, U. A., Chawla, I., and Pathak, P. M., 2020. "On determining shortest path in joint space of a cabledriven parallel robot for point-to-point motion". In 2020 28th Mediterranean Conference on Control and Automation (MED), pp. 984-989.

[29] Métillon, M., Cardou, P., Subrin, K., Charron, C., and Caro, S., 2021. "A Cable-Driven Parallel Robot With FullCircle End-Effector Rotations”. Journal of Mechanisms 
and Robotics, 13(3), 03. 031115.

[30] Lessanibahri, S., Cardou, P., and Caro, S., 2020. "A CableDriven Parallel Robot with an Embedded Tilt-Roll Wrist". Journal of Mechanisms and Robotics, 12(2), Feb.

[31] Baklouti, S., Courteille, E., Lemoine, P., and Caro, S., 2019. "Vibration Reduction of Cable-Driven Parallel Robots through Elasto-dynamic Model-Based Control". Mechanism and Machine Theory, 139, May, pp. 329-345.

[32] Ruiz, A. L. C., Caro, S., Cardou, P., and Guay, F., 2015. "Arachnis: Analysis of robots actuated by cables with handy and neat interface software". In Cable-Driven Parallel Robots, A. Pott and T. Bruckmann, eds., Springer International Publishing, pp. 293-305. 